\title{
Tumor rabdoide teratoide atípico del sistema nervioso central con INI1 positivo: presentación de un caso pediátrico
}

\section{Central nervous system atypical teratoid/ rhabdoid tumor with retained INI-1: a pediatric case}

\author{
"Alejandra Calderón-Gasca MD1* \\ " Kelly Paz MD1 2 \\ ${ }^{1}$ Instituto Nacional de Cancerología, Bogotá - Colombia. \\ ${ }^{2}$ Universidad Militar Nueva Granada
}

\section{Resumen}

Se presenta el caso de una paciente de 11 años de edad con diagnóstico de tumor rabdoide teratoide atípico del sistema nervioso central, con retención de la expresión del marcador INI 1, negatividad del marcador BRG1 y estudios de genética sin ninguna alteración correspondiente al cromosoma 22. Se logró una resección completa de la lesión, con posterior consolidación del tratamiento con quimioterapia y radioterapia y, a la fecha, en remisión de la enfermedad. La relevancia de este caso es la positividad del INI-1 en la inmunohistoquímica, que en el contexto de un tumor rabdoide teratoide atípico, no descarta esta entidad patológica, siendo útil la realización del marcador BRG1 para establecer el diagnóstico.

Palabras clave: Tumor rabdoide; teratoma; sistema nervioso central; niño; proteína SMARCB1; inmunihistoquímica; INI-1.

\section{Abstract}

We report a case of AT/RT in an 11 years old female, with a retained expression of INI1 by immunohistochemistry and loss of expression of BRG1 protein by immunohistochemistry. Genetic tests reveal no alteration related to chromosome 22. Complete resection of the lesion was performed, then she received chemotherapy and radiotherapy treatment. She is in remission 3 years following completion of treatment. This case shows that positivity for INI1 in immunohistochemistry, does not rule out a diagnosis of AT/RT, and additional testing, like BRG1 protein by immunohistochemistry, could be useful to establish the diagnosis.

Keywords: Rhabdoid tumor; teratoma; central nervous system; child; SMARCB1 protein; immunohistochemistry; INI-1.

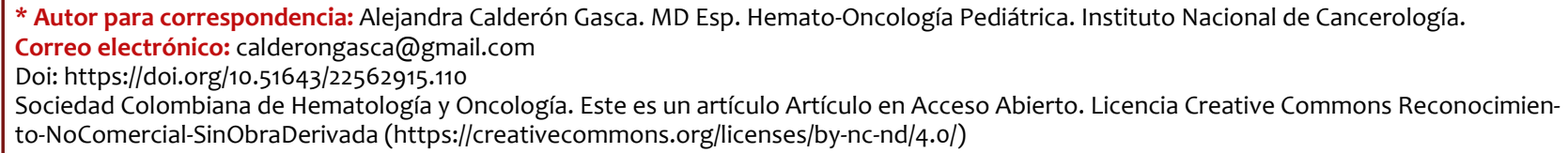




\section{Introducción}

El tumor rabdoide teratoide atípico (TRTA) del sistema nervioso central (SNC) fue descrito por primera vez por Rorke et al. en $1987^{1}$. Se trata de un tumor maligno embrionario raro del sistema nervioso central, con un pico de incidencia entre el nacimiento y los 3 años de edad. Constituye aproximadamente el 1 a $2 \%$ de los tumores pediátricos del SNC y en el $50 \%$ de los casos se localiza en la fosa posterior ${ }^{1,2,3}$. El TRTA tiene una mediana de sobrevida de 10 a 14 meses ${ }^{4,5}$.

El diagnóstico del TRTA se basa en la identificación morfológica e inmunohistoquímica de células rabdoides teratoides, las cuales muestran inmunoreactividad para un amplio rango de marcadores epiteliales, mesenquimales, gliales y neuronales ${ }^{1,6,7}$.

La pérdida de tinción de SMARCB1/SMARCA4 es un marcador que define TRTA, el SMARCB1, también es conocido como INI1, hSNF5, BAF47. Se trata de un gen supresor de tumor, localizado en 22q11.23, que hace parte de un complejo de restructuración de la cromatina dependiente de trifosfato de adenosina (ATP) con switch (SWI) y sacarosa no fermentable (SNF) 7,8,9,10,11.

En los casos de pacientes con tumores teratoides rabdoides atípicos asociados a la pérdida de SMARCB1, no es usual encontrar otras alteraciones genómicas.

Se han descrito, en menor medida, tumores sin SMARCA4 (pero que retienen SMARCB1); de ahí la relevancia de realizar marcadores de inmunohistoquímica adicionales como el BRG17,8,9,11.

\section{Caso Clínico}

Paciente de 11 años de edad con cuadro de 3 meses de cefalea y diplopía, a quien fue realizada una tomografía axial computarizada (TAC) de cráneo con contraste, documentándose una lesión intraaxial frontal derecha, con efecto de masa sobre las estructuras adyacentes de aspecto inespecífico de $4748 \times 45 \mathrm{~mm}$ y una resonancia magnética ( $R M N)$ cerebral que mostró una lesión intraaxial frontal derecha de 50 x $47 \times 44 \mathrm{~mm}$, con marcado realce del medio de contraste, áreas de degeneración quística o necrosis, y desplazamiento de la línea media, así como herniación transesfenoidal posterior derecha secundaria e incipiente infiltración leptomeníngea.

Fue valorada por el servicio de neurocirugía, y se decidió realizar resección de la lesión, lográndose una resección completa reportada por el equipo de neurocirugía, así como por la resonancia magnética cerebral de control, tomada a las 48 horas del procedimiento quirúrgico. Los estudios de patología demostraron la presencia de un tumor maligno de alto grado (WHO IV), con perfil de inmunohistoquímica que apoya diagnóstico de tumor teratoide/rabdoide atípico, con INI-1 (+) retenido, PGP9.5 (+), CKAE1AE3 $(+)$, cromogranina (-), P53 (-), neurofilamentos $(-)$, miogenina $(-)$, desmina $(+)$, vimentina $(+)$, CD99 (-), PR (-), S100 (+). Los estudios de extensión: citológico del líquido cefalorraquídeo y RMN del neuroeje, fueron negativos.

Se realizó una revisión extrainstitucional de la patología en un centro fuera del país, donde se confirmó el diagnóstico de un TRTA, con retención de la expresión de INI 1 y pérdida de la expresión del producto Brahma-related gene 1 (BRG1) del gen SMARCA4, por inmunohistoquímica. Los estudios genéticos fueron negativos para mutaciones en la línea germinal. El estudio para la deleción del cromosoma 22 fue negativo.

Se consolidó el tratamiento quirúrgico con la administración de la radioterapia externa técnica IMRT hasta 36 Gy cráneoespinal, con refuerzo al lecho hasta $54 \mathrm{GY}$. Posteriormente recibió seis bloques de quimioterapia sistémica con ICE (Ifosfamida, Carboplatino y Etopósido).

En las imágenes de fin de tratamiento y seguimiento, se documenta el estado postoperatorio de craneotomía frontal derecha con área focal de malacia y gliosis en el lóbulo frontal, sin evidencia de lesiones que sugieran residuo o recidiva tumoral.

A la fecha, más de dos años después de la terminación del tratamiento, la paciente se encuentra asintomática y sin evidencia de recaída. 


\section{Discusión}

El tumor rabdoide teratoide atípico es una patología infrecuente en la edad pediátrica; se estima entre un $1 \%$ - 2 \% de las neoplasias de alto grado del SNC ${ }^{12,13}$.

Aproximadamente un $70 \%$ de los pacientes son menores de 3 años al debut, con un predominio ligero en el sexo masculino; frecuentemente es subdiagnosticado y se confunde con otro tipo de neoplasias como el meduloblastoma, dada su localización. Aunque puede estar en todo el SNC, tiene un ligero predominio en la fosa posterior 3,14 .

La estrategia actual de tratamiento incluye la resección quirúrgica, radioterapia y quimioterapia adyuvantes y, en algunos casos, trasplante 15,16 .

En general, tiene un pronóstico sombrío que se aproxima a los 12 meses de sobrevida. Además, tiene una presentación en la que, al diagnóstico, se puede encontrar metástasis extra craneal hasta en un $40 \%$ de los casos, que puede localizarse en el hígado, pulmón, hueso, piel y ganglios linfáticos de la cabeza y cuello y, por siembra directa de catéteres de derivación ventriculoperitoneal $1,3,17$.

Su diagnóstico depende principalmente de la evaluación morfológica y la inmunotinción de algunos marcadores, como la falta expresión de la proteína INI1, que se considera altamente sensible y específica para su diagnóstico. Sin embargo, no está en el $100 \%$ de los tumores rabdoides/teratoides ${ }^{1,7}$.

En el caso que se presenta, la inmunomarcación del INI1 fue positiva y el estudio genético del cromosoma 22 negativo, pero con pérdida de la expresión del BRG1 por inmunohistoquímica, patrón que ya ha sido reportado previamente en la literatura. Por lo tanto, cabe resaltar que, aunque se conserve la expresión del INI1 (SMARCB1), no se puede descartar el diagnósti- co de ATRT y, que si se encuentra un patrón de morfología compatible con ATRT, con INI1 positivo, deben realizarse estudios adicionales para afirmar el diagnóstico.

\section{Conclusión}

El tumor rabdoide teratoide atípico es una entidad patológica infrecuente, con un pico de presentación en la edad preescolar y con una sobrevida por lo general pobre. La resección máxima segura obtenida, idealmente total, la quimioterapia intratecal y sistémica, así como la quimioterapia de altas dosis con trasplante autólogo de células progenitoras hematopoyéticas y la radioterapia, han dado lugar a resultados favorables.

La localización infrecuente, una edad fuera del rango típico y el hallazgo del INI1 retenido, con negatividad para el marcador BRG1, hacen de este caso una oportunidad para el aprendizaje, para no descartar el diagnóstico de ATRT, a pesar de la presencia del INI-1. Deben siempre agotarse los estudios disponibles para establecer el diagnóstico.

- $\quad$ Aspectos éticos: Los autores declaran que el estudio se ajusta a la Resolución 8430 de 1993; su ejecución fue aprobada y vigilada por el Comité de Ética Independiente del IDC Las Américas auna, el cual cumple con las normas de buenas prácticas clínicas en todas sus actividades.

- $\quad$ Fuente de financiación: Los autores declaran que esta investigación no ha recibido becas específicas de agencias del sector público, sector comercial o sin ánimo de lucro.

- Conflicto de interés: Los autores manifiestan no tener conflictos de intereses en este estudio. 


\section{Referencias}

1. Chou Y, Cheng S, Hsieh K, Wang C, Chen S, et al. Bacillus cereus septicemia in a patient with acute lymphoblastic leukemia: A case report and review of the literatura. J Microbiol Immunol Infect [Internet]. 2016;49(3):448-51. https://doi.org/10.1016/j.jmii.2013.06.010.

2. Yoshida M, Akiyama N, Fujita H, Miura K, Miyatake J, et al. et al. Analysis of bacteriemia/fungemia and pneumonia accompanying acute myelogenous leukemia from 1987 to 2001 in the Japan Adult Leukemia Study Group. Int J Hematol [Internet]. 2001;93(1):66-73. https://doi.org/10.1007/ s12185-010-0746-y.

3. Vodopivec I, Rinehart E, Griffin G, Johncilla M, Pecora P, et al. A Cluster of CNS Infections Due to B. cereus in the Setting of Acute Myeloid Leukemia: Neuropathology in 5 Patients. J Neuropathol Exp Neurol [Internet]. 2015;74(10): 1000-1011. https:// doi.org/10.1097/nen.0000000000000244

4. Kotiranta A, Lounatmaa K, Haapasalo M. Epidemiology and pathogenesis of Bacillus cereus infections. Microbes Infect [Internet]. 2000;2(18):189-98. https://doi.org/10.1016/s12864579(00)00269-0

5. Bottone EJ. Bacillus cereus, a volatile human pathogen. Clin Microbiol Rev [Internet]. 2010;23(19):382-98. https://doi.org/10.1128/ cmr.00073-09

6. Ramarao N, Sanchis V. The pore-forming haemolysins of Bacillus cereus: A review. Toxins (Basel) [Internet]. 2013;5:1119-39. https://dx.doi.org/10.3390\%2Ftoxins5061119

7. Hughes W, Armstrong D, Bodey G, et al. Guidelines for the use of antimicrobial agents in neutropenic patients with cancer. Clin Infect Dis [Internet]. 2002;34:730-51. https://doi.org/10.1086/339215

8. Turnbull P, Sirianni N, LeBron C, et al. MICs of se- lected antibiotics for Bacillus anthracis, Bacillus cereus, Bacillus thuringiensis, and Bacillus mycoides from a range of clinical and environmental sources as determined by the Etest. J Clin Microbiol. 2004;42:3626-34.

9. Tusgul S, Prod'hom G, Senn L, Meuli R, Bochud $P$, et al. Bacillus cereus bacteraemia: comparison between haematologic and nonhaematologic patients. New Microbe and New Infect [Internet]. 2017; 15: 65-71. https://doi.org/10.1016/j. nmni.2016.11.011

10. Denham J, Nanjappa S, Greener J. Bacillus cereus Typhlitis in a Patient with Acute Myelogenous Leukemia: A Case Report and Review of the Literature. Case Rep Infect Dis [Internet]. 2018; 2018: 7510715. https://doi.org/10.1155/2018/7510715 11. Inoue D, Nagai $Y$, Mori M, Nagano S, Takiuchi Y, et al. Fulminant sepsis caused by Bacillus cereus in patients with hematologic malignancies: Analysis of its prognosis and risk factors. Leuk Lymphoma [Internet]. 2010;51:860-69. https://doi. org/10.3109/10428191003713976

12. Gaur A, Patrick C, McCullers J, Flynn M, Pearson $\mathrm{T}$, et al. Bacillus cereus bacteremia and meningitis in immunocompromised children. Clin Infect Dis [Internet]. 2001;32: 1456-62. https://doi. org/10.1086/320154

13. Gilden D, Cohrs RJ, Mahalingam R, Nagel $M$. Varicella zoster virus vasculopathies: Diverse clinical manifestations, laboratory features, pathogenesis, and treatment. Lancet Neurol [Internet]. 2009;8:731-40. https://doi.org/10.1016/s14744422(09)70134-6

14. Ikeda M, Yagihara Y, Tatsuno K, Okazaki M, Okugawa S, Moriya K. Clinical characteristics and antimicrobial susceptibility of Bacillus cereus blood stream infections. Ann Clin Microbiol Antimicrob [Internet]. 2015;14:43. https://doi.org/10.1186/ s12941-015-0104-2 